\title{
Complementary Chassis Systems for Ground Vehicles Safety
}

\author{
Moad Kissai ${ }^{1}$, Xavier Mouton $^{2}$, Bruno Monsuez ${ }^{1}$, Didier Martinez $^{2}$, and Adriana Tapus ${ }^{1}$
}

\begin{abstract}
Redundancy of integrated systems has always been used in order to increase vehicle's safety. Some new technologies are however too expensive to be redundant. This paper proposes a new way to preserve the safety by enabling a kind of complementarity between different chassis systems. Optimization-based control allocation redistribution algorithms are used in order to find the best way to combine conflicted systems. Results have shown that one system can take over another one when a failure occurs making the control logic fault-tolerant and reconfigurable. This suits better future cars as additional chassis systems are intended to be integrated.

Index Terms-Control Allocation, Fault-Tolerance, Reconfiguration, Chassis Systems, Vehicle Dynamics, Online Optimization.
\end{abstract}

\section{INTRODUCTION}

The automotive sector is facing one of its biggest revolutions with the arrival of autonomous vehicles. Several technical challenges and ethical debates are still not completely solved, which delay their commercialization. One of the consequences of making vehicles autonomous is the growing number of smart chassis systems to integrate. For each new problem, car manufacturers and equipment suppliers propose constantly new subsystems with new features to solve the problem. It started by brake-based active systems, e.g. the Anti-lock Braking System (ABS) in 1978 [1]. Then steeringbased systems got more interest for their bigger influence on car's lateral behavior, e.g. the 4-Wheel Steering (4WS) system in 1987 [1]. So, at first, systems based on longitudinal tire forces control were used for vehicle's longitudinal control, and systems based on lateral tire forces control were used for vehicle's lateral control. With the appearance of systems such as the Electronic Stability Program (ESP) [2], the previous approach was no longer valid. This is also the case for any system that uses different right longitudinal tire forces than the left ones as the Torque Vectoring system [3]. When several subsystems control the same physical quantity, the overall system becomes over-actuated. If no coordination is ensured, conflicts may occur causing the vehicle's loss of control. A proper way to prevent this would be to formulate the problem as a constrained optimization problem. The solver can then allocate the control optimally avoiding any conflict and without exceeding any constraint.

\footnotetext{
${ }^{1}$ Moad Kissai, Bruno Monsuez, and Adriana Tapus are with ENSTA ParisTech, Department of Computer and System Engineering, 828 Boulevard des Marchaux, 91762 Palaiseau Cedex, France \{moad.kissai, bruno.monsuez, adriana.tapus\}@ensta-paristech.fr

${ }^{2}$ Xavier Mouton, and Didier Martinez are with Group Renault, Chassis Systems Department, 1 Avenue du Golf, 78280 Guyancourt, France \{xavier.mouton, didier.d.martinez\}@renault.com
}

This has already been developed in flight control, when conventional ganging methods were no longer able to handle advanced aircraft [4]. As the overall system is over-actuated, if an optimized coordination is taken into account, multiple solutions can be found. This gives additional features as ensuring multiple objectives at the same time when it is possible [5]. One of the main objectives, if it is not the most important one, is the vehicle's safety.

One common industrial practice is redundancy of safety critical systems. In [6] a redundancy-based fault detection and isolation regarding the Steer-By-Wire (SBW) has been proposed to reduce the total number of redundant road-wheel angle sensors while maintaining a high level of reliability. Authors of [7] used a switched control scheme in order to control redundant planar robotic manipulators. An example has also been given in [8] to demonstrate the effectiveness of a fault-tolerant control against partial actuator failures.

However, several expensive systems cannot be redundant in commercial ground vehicles. As different systems can influence the same physical quantity in an over-actuation context, the existing synergies between different chassis systems should be taken benefit from [9]. This can ensure some sort of complementarity between systems with different natures. So if a system fails, another completely different system can take over. Techniques used to solve the redundancy problem can be used to solve over-actuation problems. But attention should be given to the control technique to employ, as in this case, the control should switch from a system with a given behavior to another one with different behavior. For this reason, control allocation methods are more suitable, as in contrast to the existing reconfiguration methods that modify controllers to tolerate faults, control allocation methods only change allocation laws. In one hand, this is advantageous in a way that allocation laws take account of systems with different nature, and in the other hand, this implies that stability of the closed-loop system after reconfiguration can be guaranteed [10].

This paper outlines the benefits of making complementary non-redundant chassis systems to enhance vehicle's safety. A simple new example of a vehicle equipped by the Active Rear Steering (ARS) system and the brake-based Vehicle Dynamics Control (VDC) system is provided in order to demonstrate the effectiveness of the proposed method. The architecture proposed is extensible to additional subsystems and additional objectives, which makes it a good candidate for future autonomous vehicles [5]. Additional objectives could include energy consumption [11] or tire energy dissipation [12]. Good results were obtained using Matlab/Simulink ${ }^{(}$ co-simulated with AMESim $^{\circledR}$, which contains high-fidelity 
vehicle models to provide more realistic signals. Results showed that one system can easily complete the maneuver of the other one, proving that there is no need of redundancy of such expensive system such as the ARS system.

The rest of the paper is structured as follows: We start in Section II by providing the dynamics modelings necessary for the high-level control. In Section III, the method used reallocate the control commands in case of failure is detailed. Section IV presents results obtained by co-simulation of Matlab/Simulink ${ }^{\circledR}$ and AMESim ${ }^{\circledR}$. A discussion about the relevance and implication of this method is provided in Section V. Conclusions and future works are outlined in Section VI.

\section{Vehicle Dynamics CONTROL}

In order to show the effectiveness and relevance of the method presented in this paper, we choose to give a practical example of one of today's commercial vehicles. This should not be seen as a particular situation. In Section III, a general explanation of the method is provided. This can be adaptable to any situation. The vehicle chosen in this paper is the latest Renault Talisman ${ }^{1}$. This vehicle is equipped with the Active Rear Steering (ARS) system, which has a strong influence on the group's brand image, and the brake-based Vehicle Dynamics Control (VDC) system, which functionality is similar to that of the ESP. We chose these two systems because they both influence the vehicle's yaw rate. Moreover, the ARS system produces a rear steering angle generating a lateral tire force, while the VDC system generates a yaw moment by means of differential longitudinal tire forces between right tires and left tires. This makes the two systems having complete different behaviors. In this situation, industries tend to avoid operating both conflicting systems in the same time, making the coordination downstream the systems by enabling one command at a time [5]. In this work, an upstream approach is adopted that enables combining both systems and making benefit from the vehicle's whole potential.

\section{A. System Modeling}

Unlike most of lateral control papers that use the bicycle model [13]-[16], here a four-wheeled vehicle model is chosen (more details are provided in the following subsections). This choice is motivated by the fact that we want to allocate optimally longitudinal tire forces to each tire by means of the VDC system, and to do so, we need to know the effect of each tire on the overall vehicle dynamics.

1) Vehicle model: Fig. 1 illustrates the model adopted. As only longitudinal and lateral forces are controllable, vertical motions were ignored making the model planar. However, vertical forces variations have significant influence on tire behavior [17],[18]. This should be taken into account in the tire model.

\footnotetext{
${ }^{1}$ We thank the Group Renault for sharing its technical parameters.
}

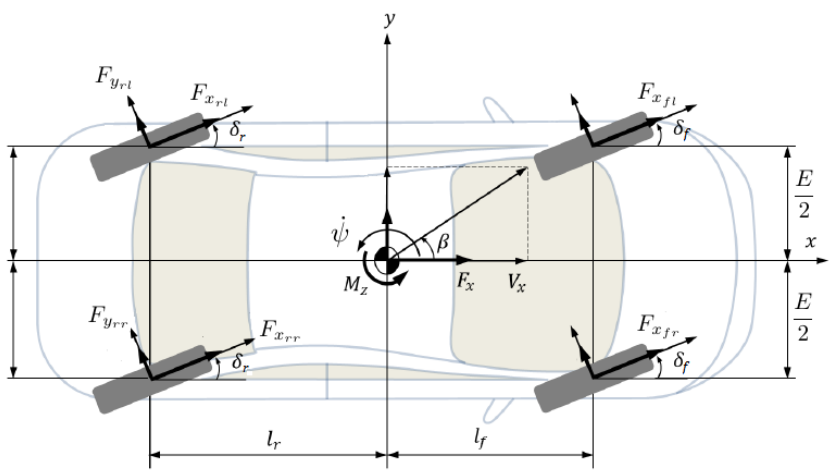

Fig. 1. The four-wheeled planar vehicle model (adapted from [14]).

Using Newton's laws of motion, we find the following state-space representation:

$$
\left\{\begin{array}{l}
\dot{x}(t)=A(t) x(t)+B u(t) \\
y(t)=C x(t)
\end{array}\right.
$$

Where:

$$
\begin{array}{ll}
\text { - } x(t)=\left[\begin{array}{c}
V_{x} \\
\dot{\psi}
\end{array}\right] & : \text { is the state vector, } \\
\text { - } y(t)=\left[\begin{array}{c}
V_{x} \\
\dot{\psi}
\end{array}\right] & : \text { is the output vector, } \\
\text { - } u(t)=\left[\begin{array}{c}
F_{x_{t o t}} \\
M_{z_{t o t}}
\end{array}\right] & : \text { is the input vector, } \\
\text { - } A(t)=\left[\begin{array}{ll}
0 & V_{y} \\
0 & 0
\end{array}\right] & : \text { is the state matrix, } \\
\text { - } B=\left[\begin{array}{ll}
\frac{1}{M} & 0 \\
0 & \frac{1}{J_{z}}
\end{array}\right] & : \text { is the input matrix, } \\
\text { - } C=\left[\begin{array}{ll}
1 & 0 \\
0 & 1
\end{array}\right] & : \text { is the output matrix. }
\end{array}
$$

With:

$$
\left\{\begin{aligned}
F_{x_{t o t}}= & \left(F_{x_{f l}}+F_{x_{f r}}\right) \cos \left(\delta_{f}\right)+F_{x_{r l}}+F_{x_{r r}} \\
M_{z_{t o t}}= & \left(F_{x_{f l}}+F_{x_{f r}}\right) l_{f} \sin \left(\delta_{f}\right) \\
& +\left(F_{x_{f r}}-F_{x_{f l}}\right) \frac{E}{2} \cos \left(\delta_{f}\right) \\
& +\left(F_{x_{r r}}-F_{x_{r l}}\right) \frac{E}{2}-F_{y_{r}} l_{r}
\end{aligned}\right.
$$

Where:

- $V_{x} \quad$ : longitudinal velocity of the vehicle,

- $V_{y} \quad$ : lateral velocity of the vehicle,

- $\dot{\psi} \quad$ : yaw rate of the vehicle,

- $M \quad$ : vehicle's mass,

- $J_{z}$ : vehicle's yaw moment of inertia,

- $F_{x_{f, r}}$ : front-right braking force,

- $F_{x_{f, l}}$ : front-left braking force,

- $F_{x_{r, r}}$ : rear-right braking force,

- $F_{x_{r, l}}$ : rear-left braking force,

- $F_{y_{r}}=F_{y_{r l}}+F_{y_{r r}}$ lateral rear force, 
- $\delta_{f} \quad$ : front steering angle,

- $l_{f} \quad$ : distance between the front axle and the vehicle's center of gravity $(\mathrm{CoG})$,

- $l_{r} \quad$ : distance between the rear axle and the vehicle's $\mathrm{CoG}$

- $E \quad$ : vehicle's track.

Note that we have considered only controllable forces to distribute.

2) Tire model: To complete modeling the system, a tire model is required. As long as systems with different behaviors are concerned soliciting tires both longitudinally and laterally, a combined tire model is needed. This model should be precise enough in stable operating points, but also simple enough to be used in real-time control maneuvers.

Therefore, the linear tire model with varying parameters is chosen, which details and validation through AMESim ${ }^{\circledR}$ is provided in [19]. We recall here the main results. Tire forces are expressed using varying stiffnesses, taking into account couplings, vertical forces influence, and friction influence.

$$
\left\{\begin{array}{l}
F_{x}=C_{s}^{*}\left(\alpha, \mu, F_{z}\right) \kappa \\
F_{y}=C_{\alpha}^{*}\left(\kappa, \mu, F_{z}\right) \alpha
\end{array}\right.
$$

where:

- $\kappa \quad:$ the longitudinal slip,

- $\alpha \quad$ : the side-slip,

- $\mu \quad:$ the friction coefficient,

- $F_{z} \quad:$ the vertical load,

- $C_{s}^{*}\left(\alpha, \mu, F_{z}\right)$ : the tire varying longitudinal stiffness with respect to the side-slip $\alpha, \mu$, and $F_{z}$,

- $C_{\alpha}^{*}\left(\kappa, \mu, F_{z}\right)$ : the tire varying cornering stiffness with respect to the longitudinal slip $\kappa, \mu$, and $F_{z}$.

Detailed expressions of $C_{s}^{*}\left(\alpha, \mu, F_{z}\right)$ and $C_{\alpha}^{*}\left(\kappa, \mu, F_{z}\right)$ can be found in [19].

In order to respect the friction ellipse concept described in [17], which describes penalization between tire forces, dynamic constraints are added [19]:

$$
\left\{\begin{array}{l}
F_{x} \leq \sqrt{\left(\mu F_{z}\right)^{2}-F_{y}^{2}} \\
F_{y} \leq \sqrt{\left(\mu F_{z}\right)^{2}-F_{x}^{2}}
\end{array}\right.
$$

\section{B. High-Level Control}

In order to distribute the forces to each effector, the generalized force $F_{x_{t o t}}$ and moment $M_{z_{t o t}}$ should be first generated. According to equations (1) and (2), a multivariable high-level controller is needed. Due to expression of the state matrix $A(t)$, couplings between the longitudinal velocity and the yaw rate are related to the lateral velocity value. $V_{y}$ can be considered as a varying parameter. The vehicle model is then linearized with respect to a range of $V_{y}$ values. Moreover, as several parametric uncertainties exist at the vehicle level ${ }^{2}$ and the tire level ${ }^{3}$, a robust controller should be designed. For these reasons, a Gain-Scheduled $H_{\infty}$ controller has been chosen to ensure the high-level control

\footnotetext{
${ }^{2}$ Mass, moment of inertie, and so on.

${ }^{3}$ Basically the friction coefficient
}

where $V_{y}$ is the scheduling parameter. As the scope of this paper is not the high-level vehicle dynamics control, the design of this controller is not addressed here.

\section{FAULT-TOLERANCE USING CONTROL REALLOCATION}

Once the generalized forces required to ensure safe vehicle motions are calculated, they should be optimally distributed to the different systems in order to effectively generate them. This upstream approach has a lot of advantages as extensibility, modularity, and easy design of fault-tolerance control [5]. This separation of high-level control and control allocation allows taking into account the actuators constraints and states in an explicit way. So if an actuator fails, only the allocation will be updated. The high-level control remains unchanged, and the stability of the overall system can still be guaranteed.

\section{A. Method Description}

The control allocation problem can be defined as follows [26]: find the control vector, $\vec{u} \in \mathbb{R}^{n}$ such that

$$
\mathbf{B} \vec{u}=\vec{v}
$$

subject to

$$
\left\{\begin{array}{r}
\vec{u}_{\min } \leq \vec{u} \leq \vec{u}_{\max } \\
\dot{\vec{u}} \leq \dot{\vec{u}}_{\max }
\end{array}\right.
$$

where $\mathbf{B} \in \mathbb{R}^{m \times n}$ is a control effectiveness matrix, $\vec{u}_{\text {min }} \in$ $\mathbb{R}^{n}$ and $\vec{u}_{\text {max }} \in \mathbb{R}^{n}$ are the lower and upper position limits, respectively, $\dot{\vec{u}} \in \mathbb{R}^{n}$ is the control rate, $\dot{\vec{u}}_{\max } \in \mathbb{R}^{n}$ is the maximum control rate, $\vec{v} \in \mathbb{R}^{m}$ are the desired accelerations, $n$ is the number of control effectors, and $m$ is the number of axes to control with $n>m$.

The allocation is closely related to the effectiveness matrix B. It expresses each control component's effectiveness to modify the overall behavior of the system. The idea is to simply modify this matrix to take account of the actuators states. In this way, if an actuator fails, the value of the effectiveness matrix will change to express the fact that the failed actuator has no longer effect on the overall system. The control will be then reallocated to the healthy actuators continuing the maneuver in a degraded mode.

\section{B. Application}

For the ARS-VDC coordination case, the control vector contains controllable tire forces:

$$
\vec{u}=\left[\begin{array}{c}
F_{x_{f l}} \\
F_{x_{f r}} \\
F_{x_{r l}} \\
F_{x_{r r}} \\
F_{y_{r}}
\end{array}\right]
$$

$\vec{u}_{\min }$ and $\vec{u}_{\max }$ reflect tire limits with respect to the friction ellipse concept (7) and (8). We suppose that the friction coefficient can be estimated [21], and so can be the vertical 
loads [14]. The desired acceleration $\vec{v}$ contains in this case the generalized forces:

$$
\vec{v}=\left[\begin{array}{l}
F_{x_{t o t}} \\
M_{z_{t o t}}
\end{array}\right]
$$

To complete the problem definition described in (9), $\mathbf{B}$ is filled by geometric relations between the vehicle and its tires using equations (3) and (4):

$$
\mathbf{B}=\left[\begin{array}{ccccc}
\cos \left(\delta_{f}\right) & \cos \left(\delta_{f}\right) & 1 & 1 & 0 \\
b_{2,1} & b_{2,2} & -\frac{E}{2} & \frac{E}{2} & -l_{r}
\end{array}\right]
$$

where:

$$
\begin{aligned}
& \text { - } b_{2,1}=l_{f} \sin \left(\delta_{f}\right)-\frac{E}{2} \cos \left(\delta_{f}\right), \\
& \text { - } b_{2,2}=l_{f} \sin \left(\delta_{f}\right)+\frac{E}{2} \cos \left(\delta_{f}\right) .
\end{aligned}
$$

In this paper, we suppose that we are able to detect and isolate the fault ${ }^{4}$. Moreover, we recall that our objective is to control the vehicle's yaw rate by means of two conflicted chassis systems. We modify then only the second line of the effectiveness matrix to take into account systems' failures. Let us note $\varphi_{i}$ the flag of the $i^{t h}$ actuator expressing its status where:

$$
\varphi_{i}= \begin{cases}1 & \text { if the actuator is working fine } \\ 0 & \text { otherwise }\end{cases}
$$

The effectiveness matrix becomes then:

$$
\mathbf{B}=\left[\begin{array}{ccccc}
\cos \left(\delta_{f}\right) & \cos \left(\delta_{f}\right) & 1 & 1 & 0 \\
b_{2,1} \varphi_{1} & b_{2,2} \varphi_{2} & -\frac{E}{2} \varphi_{3} & \frac{E}{2} \varphi_{4} & -l_{r} \varphi_{5}
\end{array}\right]
$$

\section{Optimization Algorithm Choice}

Two main criteria should be taken into account. First, the algorithm should provide enough potential to solve the control allocation and reallocation and converge to the optimum in a finite number of iterations. Secondly, the algorithm should be fast enough to be suitable for real-time operations.

Various techniques have been tested. In [14], the DaisyChain method has been combined with the Weighted PseudoInverse (WPI) method to handle coordination of the ESP with the Electric Power-Assisted Steering (EPAS) system. The advantage of this technique is the fact that it is based on an algebraic calculation which makes it faster with respect to online optimization based methods. However, on one hand, the Daisy-Chain method imposes a predefined prioritization of one system over another, which contradicts an upstream approach necessary for future cars [5]. And on the other hand, the WPI is more suitable as long as only one objective is pursued. For multi-objectives problems, no algebraic expression exists. This limits the utilization of this method.

In this work, we focus more on online optimization techniques. Active Set Algorithms (ASA) have shown good

\footnotetext{
${ }^{4}$ Renault's smart actuators for both systems contain fault detection modules.
}

results in this context. Here, the optimal control vector is expressed as [22]:

$$
\vec{u}_{\text {opt }}=\arg \min _{\vec{u}_{\min } \leq \vec{u} \leq \vec{u}_{\max }}\|\mathbf{A} \vec{u}-\vec{b}\|
$$

Two different methods based on ASA have been derived. Sequential Least Squares (SLS) uses two stage ASA to separate the global problem into two optimization problems [22]. This leads to the following formulation:

$$
\left\{\begin{aligned}
\vec{u}_{o p t} & =\arg \min _{\vec{u} \in \Omega}\left\|\mathbf{W}_{\mathbf{u}}\left(\vec{u}-\vec{u}_{p}\right)\right\| \\
\Omega & =\arg \min _{\vec{u}_{\min } \leq \vec{u} \leq \vec{u}_{\max }}\left\|\mathbf{W}_{\mathbf{v}}(\mathbf{B} \vec{u}-\vec{v})\right\|
\end{aligned}\right.
$$

Where:

- $\vec{u}_{p} \quad$ : preferred control vector,

- $\mathbf{W}_{\mathbf{u}}$ : non-singular weighting matrix affecting control distribution among the actuators,

- $\mathbf{W}_{\mathbf{v}}$ : non-singular weighting matrix affecting the prioritization among the virtual control components when $\mathbf{B} \vec{u}=\vec{v}$ cannot be attained due to the actuator constraints.

In contrast, the Weighted Least Squares (WLS) solves the global problem in one stage ASA by means of different weights to determine the importance of each objective [22]. Generally, the allocation objective is prioritized using a highvalue weight of the order $\gamma \simeq 10^{6}$ [22]. This gives the following expression:

$$
\begin{aligned}
\vec{u}_{\text {opt }}=\arg \min _{\vec{u}_{\min } \leq \vec{u} \leq \vec{u}_{\max }} & \left\|\mathbf{W}_{\mathbf{u}}\left(\vec{u}-\vec{u}_{p}\right)\right\|^{2} \\
& +\gamma\left\|\mathbf{W}_{\mathbf{v}}(\mathbf{B} \vec{u}-\vec{v})\right\|^{2}
\end{aligned}
$$

Other techniques non based on ASA exist as the Interior Point (IP) solver [23] and Cascading Generalized Inverses (CGI) [24]. One of the most interesting one is the Fixed-Point Iteration (FXP) [25] because of its rapidity with respecting to other optimization techniques. These techniques are compared in Section IV trough co-simulation. The reader can refer to [4],[22],[26] for further details on solver algorithms and their comparison.

\section{Low-Level Control}

The allocation algorithm generates tire forces commands. These commands should be transformed before being fed to the different actuators actuators. As the relations between efforts and actuators inputs are dynamic, a proper way to interpret the tire forces commands would be to add additional low-level controllers. However, this will add additional time lags to the global control logic. Moreover, for good precision and robustness, a closed-loop will be needed. Unfortunately, tire forces are not measurable in commercial vehicles. Therefore, here only an algebraic transformation is added:

$$
\left\{\begin{aligned}
T_{b_{i j}} & =-R_{i} F_{x_{i j}} \\
\delta_{r} & =\frac{F_{y_{r}}}{C_{\alpha}^{*}}-\left(\frac{J_{z}}{M l_{r}}-l_{r}\right) \frac{\dot{\psi}}{V_{x}}
\end{aligned}\right.
$$

Where: 
- $T_{b_{i, j}}$ : brake torque at the $i-j^{5}$ wheel,

- $R_{i}$ : wheels' effective radius of axle " $i$ ",

- $\delta_{r} \quad$ : rear steer angle.

The minus sign in equation (25) is due to the fact that the brake pressure should be positive, while the brake forces are negative. Equation (26) comes from a combination of (1),(6) with the following:

$$
\alpha_{r}=\delta_{r}-\frac{V_{y}-\dot{\psi} l_{r}}{V_{x}}
$$

Note that the new tire model is not only used in control allocation, but also in the rear steering angle computation. The value of this angle will be then adapted to respect the rear tires conditions.

\section{CO-SIMULATION RESULTS}

To give reliable results, control algorithms have been written in Matlab $^{\complement}$, while a high-fidelity vehicle model ${ }^{6}$ equipped with an ARS and VDC systems has been developed in AMESim ${ }^{\circledR}$. Simulink ${ }^{\circledR}$ is used as a bridge to co-simulate Matlab's high performance algorithms and AMESim's high fidelity vehicle model.

\section{A. Maneuver Description}

For safety tests, we choose the ISO 3888-1:1999(E) Double Lane-Change, or what is commonly called the VDA test, to validate the control logic. Fig. 2 illustrates this severe double lane-change maneuver which consists on rapidly driving a vehicle from its initial lane to another parallel lane, and returning to the initial lane, without exceeding lane boundaries. Track dimensions are mentioned in Table I.

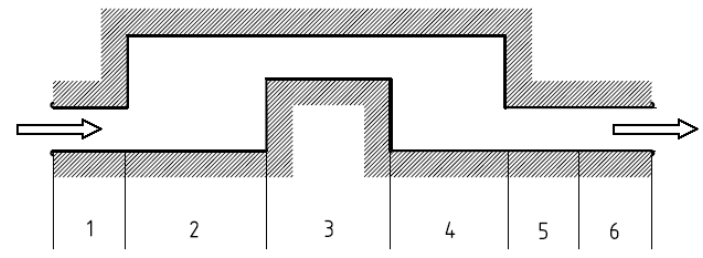

Fig. 2. Double lane-change track.

TABLE I

DOUBLE LANE-CHANGE DIMENSIONS OF SECTIONS

\begin{tabular}{|c||c||c||c|}
\hline Section & Length & Lane offset & Width \\
\hline 1 & 15 & - & $1,1 \times$ vehcile width +0.25 \\
2 & 30 & - & - \\
3 & 25 & 3.5 & $1,2 \times$ vehcile width +0.25 \\
4 & 25 & - & - \\
5 & 15 & - & $1,3 \times$ vehcile width +0.25 \\
6 & 15 & - & $1,3 \times$ vehcile width +0.25 \\
\hline
\end{tabular}

According to the ISO 3888-1:1999(E) standard, the desired speed during all the maneuver should be maintained to $80 \mathrm{~km} / \mathrm{h}$. The yaw rate target is generated using the static expression of the bicycle model [13] for fast computation.

\footnotetext{
${ }^{5} i=f$ for "front" or $r$ for "rear", and $j=l$ for "left" or $r$ for "right".

${ }^{6}$ The model has been provided by the Group Renault. Its details cannot be shared unfortunately.
}

\section{B. Results}

In order to test the effectiveness of the method proposed in Section III, three tests are carried out: comparison of the classical control allocation method with the method proposed in this paper when there is no failure, comparison of the two methods when the ARS system fails in the middle of the lane-change maneuver, and comparison of classical control allocation solvers when failure.

1) Comparison of methods when no failure: In order to focus on the method used only, the WLS algorithm is used for both methods at first. A discussion about algorithms is provided at the end of this section. Here, the VDA test is carried out normally without any failure. Fig. 3 shows the yaw rate response following both methods.

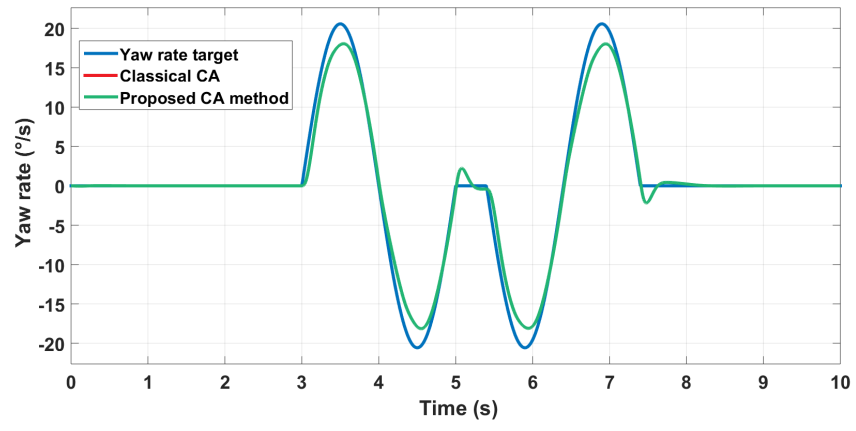

Fig. 3. Comparison of the proposed method and the classical one when no failure.

As expected, both methods exhibit the same performance as the value of the flags, $\varphi_{i}$ remains equal to 1 during all the maneuver. The effectiveness matrix for both methods is the same. This simulation serves actually to evaluate how each system is solicited. Fig. 4 shows the ARS actuation and Fig. 5 shows the VDC one.

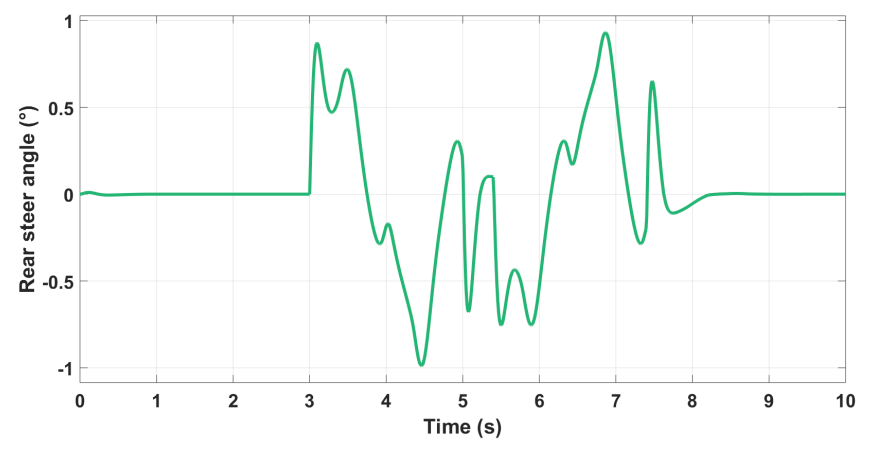

Fig. 4. Rear steer angle when no failure.

We can see that the difference between right brakes and left ones can be ignored because of their low values. The ARS system is then automatically prioritized. This could have been predicted as the yaw moment generated by the ARS system is related to the distance between the rear axle and the vehicle's CoG: $l_{r}$, which is bigger than the half value of the vehicle's track $E$ to what the VDC system is related. The ARS system is more effective than the VDC system. 


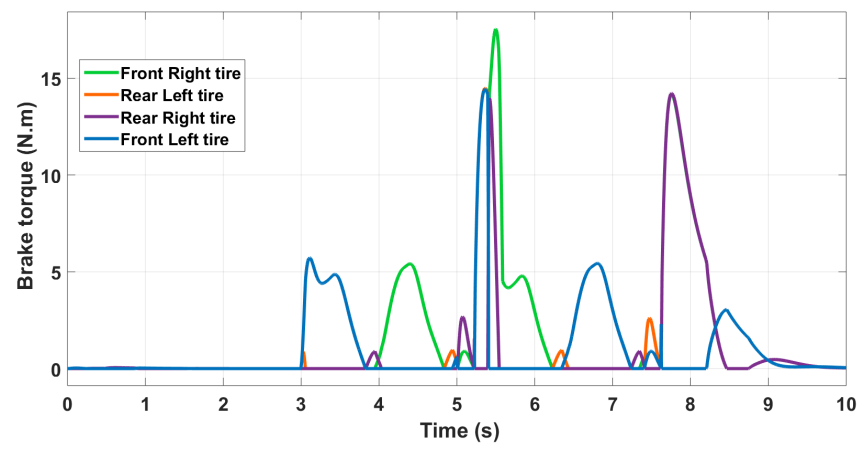

Fig. 5. VDC activation when no failure.

2) Comparison of methods when failure: As the ARS system is naturally prioritized in this maneuver, making the VDC fail will not give any pertinent result. Therefore, we choose to simulate the ARS failure at time $t=5 \mathrm{~s}$ as Fig. 6 shows. Both methods are again compared using the WLS

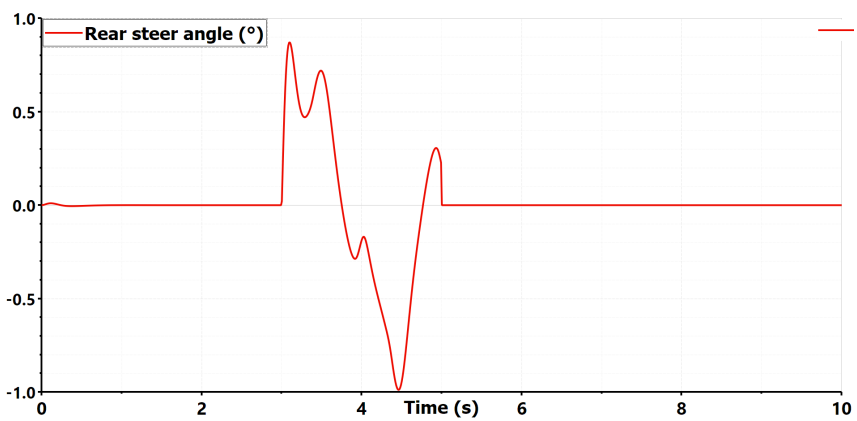

Fig. 6. Rear steer angle when ARS fails.

algorithm. The yaw rate response is illustrated in Fig. 7 . According to Fig. 7, the proposed method gives nearly the

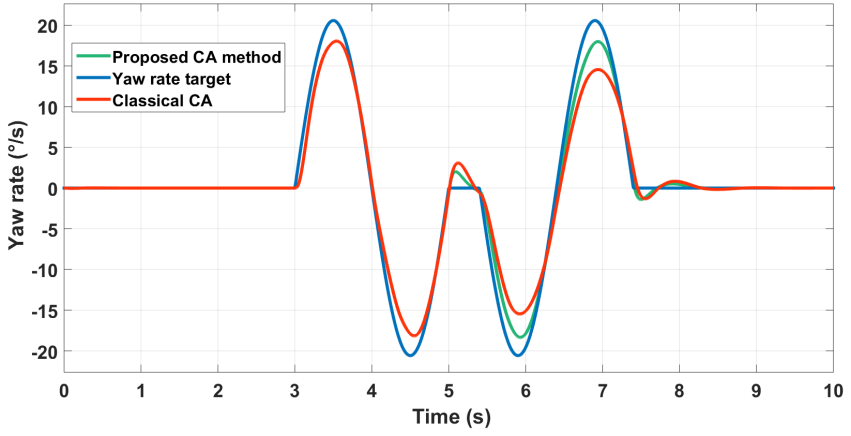

Fig. 7. Comparison of the proposed method and the classical one when ARS fails.

exact same result as the previous case when no failure has occurred, making the failure unnoticeable to the driver. In contrast, the classical control allocation method loses its precision after the failure. This is due to the fact that in the proposed method the effectiveness matrix is updated to best represent the new situation, while this is ignored in classical methods. Consequently, in the proposed method, the VDC system is more solicited to complete the maneuver that the
ARS system should have ensured. This makes both systems complementary as Fig. 8 shows.

In the classical method, the VDC is poorly utilized only because a large yaw rate error is sensed.

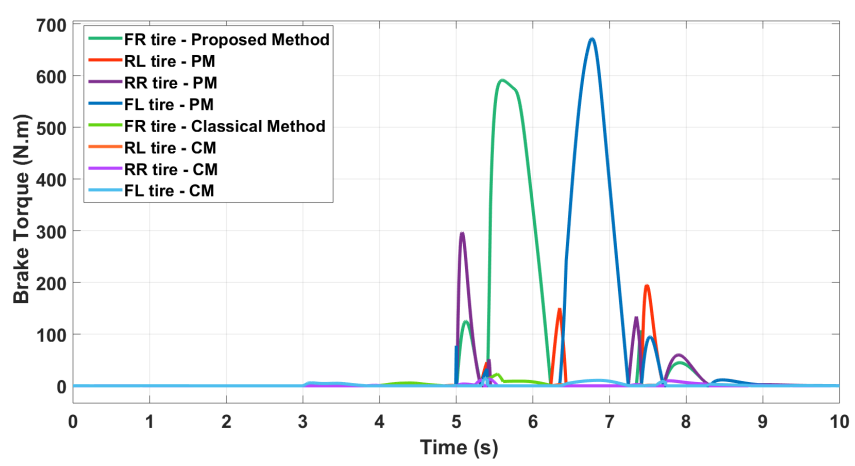

Fig. 8. Comparison of the VDC activation in the proposed method and the classical one when ARS fails.

3) Comparison of control allocation solvers: Other algorithms are compared in order to justify the utilization of each one. The Sequential Least Squares (SLS) algorithm, The Weighted Least Squares (WLS) algorithm, the Interior Point (IP) algorithm, the Fixed-Point (FXP) iteration, and the Cascading Generalized Inverses (CGI) are compared using a CORE i7 $7^{\text {th }}$ generation machine in the failure co-simulation case by means of LMS Imagine.Lab $15^{\circledR}$ and MATLAB $\mathrm{R} 2015 \mathrm{a}^{\circledR}$. Results in terms of the yaw rate response are shown in Fig. 9.

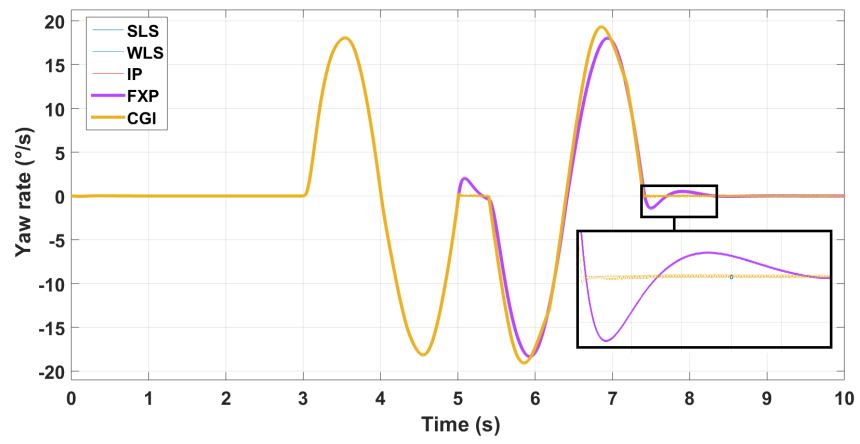

Fig. 9. Comparison of CA algorithms one when ARS fails.

SLS, WLS, IP, and FXP algorithms exhibit almost the same response. The CGI seems to be more precise. However, when zooming in Fig. 9, we can notice that the CGI signal is vibrating. This is due to non-continuous activation of the brake as Fig. 10 shows. Redistributing Pseudo-Inverses could lead to tire wear more rapidly with respect to other algorithms. Imposing some rate limits may even give different results. In fact, the CGI requires only a finite number of iterations but does not guarantee that the optimal solution is found [24].

Regarding other algorithms, simulation time may be used to select the best algorithm for real-time maneuvers. Tic/toc commands have been used for comparison purposes only. 


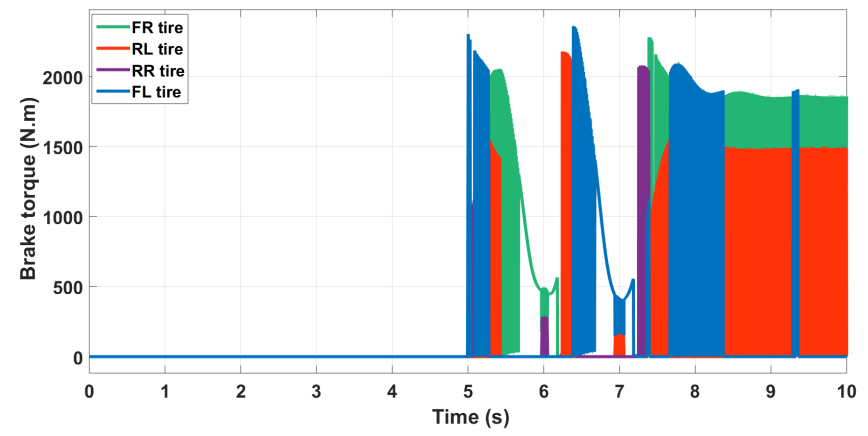

Fig. 10. VDC activation using CGI algorithm when ARS fails.

The SLS algorithm simulation completed in $175 \mathrm{~s}$, WLS in 90s, IP in 243s, FXP in 75s, and CGI in 110s. The WLS and FXP algorithms seems to be the fastest thanks to their nonsequential formulation. The WLS algorithm gives the option of easily taking into account the multi-objectives problems. This makes the WLS algorithm more attractive.

\section{CONTROL ARCHITECTURE RELEVANCE}

Integrated systems are increasing in number in order to make autonomous driving a reality. For economic competition matters, car manufacturers tend to diversify their suppliers [5]. Each system is then developed independently by equipment suppliers without taking into account the interactions with other systems. Coordination strategies should be then designed by car manufacturers to avoid conflicts and take advantage of the whole potential of the vehicle. In [5], it was concluded that the multi-layer architecture separating the high-level control from the interactions management layer is more suited for over-actuated systems.

In this way, optimization-based methods can be used giving an optimal coordination strategy. This induces several advantages.

\section{A. Safety Improvement}

Making subsystems complementary has the important advantage of improving safety. As it is shown in this paper, fault-tolerance can be ensured by simply modifying the effectiveness matrix in order to take into account the actuators' state. When a fault occurs, the value of the effectiveness matrix is updated by representing the new situation. The control is then redistributed favoring healthy actuators. The simple example provided in this work proves the effectiveness of this method in severe maneuvers as the double lane change.

In this work, actuators' states were limited to faulty or healthy. However, the failure model can be classified into four different types [10]: Lock-In-Place (LIP), Hard-Over Failure, Float, and Loss Of Effectiveness (LOF). While LIP, HOF, Float failures can be considered as total failures to their independence from input commands, the LOE failure is rather a degradation of performance with respect of nominal input due to for example actuators' aging. These cases can also be taken into account by making the parameters $\varphi_{i}$ variable with respect to the actuator performance. However, aging should be quantified by evaluating the performance degradation. More investigations are needed for addressing this aspect.

\section{B. Economic Advantage}

Complementarity of systems could reduce considerably the overall cost. In fact, smart actuators and new technologies are expensive. If autonomous vehicles rely on such systems, and if no complementarity is proposed, these systems should be redundant for safety requirements. Unlike this common practice, making integrated systems act as a backup for different other critical systems could reduce of necessary redundant systems. A deep safety study should however be carried out before taking any decision for commercial cars.

Moreover, information contained in one system can be used to reduce the number of sensors and reduce computational overheads by not duplicating controller computations [13]. Calculators hosting online optimization algorithms may be more expensive, but the number of Electronic Cuntrol Units (ECU) may be reduced [27].

\section{Potential Expansion}

Actuators' faults and constraints are not the only reason for control allocation redistribution. Ground vehicles' performance is closely related to road friction. Coefficient of friction sudden changes are the cause of many accidents [28]. In fact, even if it is said in the police jargon that $90 \%$ of accidents are due to driver errors, $50 \%$ of these accidents are actually due to the environment [29]. Normal drivers' experience is limited largely to driving well within the physical limit of adhesion, and this should be taken into account. Advanced Driver Assisted Systems should be then effective beyond this limit. Redistribution of control allocation could be used to face this problem. Using the tire model implemented in this work [5], loss of potential due to friction change can be estimated. Weights related to the maximum potential of each tire can be added to the parameters $\varphi_{i}$. In this way, if a tire approaches saturation, weights will be decreased to near zero making the control redistributed to other non-saturated tires. Consequently, the overall potential of the four tires can be used by the different integrated systems expanding the vehicle performance as Fig. 11 shows. One challenge still remains, which is the coefficient friction estimation. An ongoing work is carried out in order to overcome this challenge by means of closedloop observation techniques.

Another reason why autonomous vehicles stakeholders should favor this approach, is the ability of solving multiobjectives problems. As it was shown in [31], control allocation is able of not only preserving stability, but also favoring actuators with the least impact on energy consumption, which is very attractive for electric vehicles. This approach can also be used to generate different motion feelings. This is particularly very important for autonomous vehicles, because the challenge is not only following automatically a trajectory, but also how to follow it and which dynamics should the car favor. As motion feelings are related to vehicle's 


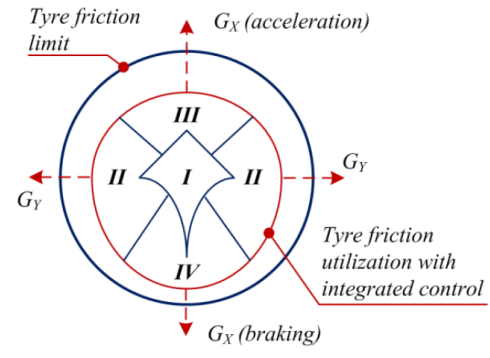

Fig. 11. Influence of integrated systems coordination on tyre friction utilization. Regions: I - without control; II - integration of active suspension and Active Front Steering (AFS) and ARS; III - integration of traction control and AFS and ARS; IV - integration of ABS and AFS and ARS [30].

accelerations according to ISO 2631-1, control allocation can be executed by combining forces respecting this standards and generating more expected motions to make drivers trust their vehicles.

\section{CONCLUSIONS}

More evidence of the necessity of employing control allocation methods have been provided in this paper. The faulttolerance criterion has been focused on to prove the safety improvement provided by control reallocation algorithms. Co-simulation results showed that by making complementary completely different chassis systems, one system can take over another one even in severe conditions, avoiding redundancy of the same system. This gives the possibility of integrating additional systems without costs concerns and accelerating autonomous vehicles development.

Real experiments should however be conducted in order to validate this approach. Close collaboration of car manufacturers is expected in order to provide a standardization of these solutions. Upcoming future works include experimental studies, multi-objectives problems, and various techniques comparison for both high-level and low-level control.

\section{REFERENCES}

[1] M. Gerard and E.Y. Lopes, Global Chassis Control and Braking Control using Tyre Forces Measurement, TU Delft, 2011.

[2] E. Liebemann, K. Meder, J. Schuh, and G. Nenninger, Safety and performance enhancement: The bosch Electronic Stability Control (ESP), SAE Technical Paper 2004-21-0060, 2004.

[3] M. Velardocchia and A. Vigliani, Control systems integration for enhanced vehicle dynamics, The Open Mech. Eng. J., vol. 7, 2013, pp. 58-69.

[4] Marc Bodson, Evaluation of Optimization Methods for Control Allocation, Journal of Guidance, Control, and Dynamics, Vol. 25, No. 4 (2002), pp. 703-711.

[5] M. Kissai, B. Monsuez and A. Tapus, Review of integrated vehicle dynamics control architectures, 2017 European Conference on Mobile Robots (ECMR), Paris, 2017, pp. 1-8.

[6] S. Anwar and L. Chen, An Analytical Redundancy-Based Fault Detection and Isolation Algorithm for a Road-Wheel Control Subsystem in a Steer-By-Wire System, in IEEE Transactions on Vehicular Technology, vol. 56, no. 5, pp. 2859-2869, Sept. 2007.

[7] B. E. Bishop and M. W. Spong, Control of redundant manipulators using logic-based switching, Proceedings of the 37th IEEE Conference on Decision and Control (Cat. No.98CH36171), Tampa, FL, 1998, pp. 1488-1493 vol.2.

[8] Y. M. Zhang and J. Jiang, Active fault-tolerant control system against partial actuator failures, in IEE Proceedings - Control Theory and Applications, vol. 149, no. 1, pp. 95-104, Jan 2002.
[9] E. Coelingh, P. Chaumette and M. Andersson, Open-interface definitions for automotive systems - Application to a Brake by Wire System, SAE 2002 World Congress, 2002-01-0267.

[10] I. Yang, D. Kim and D. Lee, Fault-tolerant control strategy based on control allocation using smart actuators, 2010 Conference on Control and Fault-Tolerant Systems (SysTol), Nice, 2010, pp. 377-381.

[11] Y. Chen and J. Wang, Energy-efficient control allocation with applications on planar motion control of electric ground vehicles, Proceedings of the 2011 American Control Conference, San Francisco, CA, 2011, pp. 2719-2724.

[12] B. Shyrokau, D. Wang, D. Savitski, K. Hoepping, V. Ivanov, Vehicle motion control with subsystem prioritization, In Mechatronics, Vol. 30, 2015, pp. 297-315.

[13] M. A. Selby, Intelligent Vehicle Motion Control, PhD thesis, University of Leeds, Feb. 2003.

[14] A. Soltani, Low Cost Integration of Electric Power-Assisted Steering (EPAS) with Enhanced Stability Program (ESP), PhD thesis, Cranfield University, 2014.

[15] L. Xiong and Z. Yu, Research on Robust Control for longitudinal Impact of 4 Wheel-Drive Hybrid Electric Vehicle, Procedia Engineering, vol. 15 , pp. 293-297, 2011.

[16] T. Bchle, K. Graichen, M. Buchholz, and K. Dietmayer, SlipConstrained Model Predictive Control Allocation for an All-Wheel Driven Electric Vehicle, IFAC Proceedings Volumes, vol. 47, issue 3 , pp. 12042-12047, 2014.

[17] H.B. Pacejka, Tyre and Vehicle Dynamics, Second Edition. Elsevier, Butterworth-Heinemann, 2006.

[18] H. Dugoff, P. Fancher and L. Segel, "Tire Performance Characteristics Affecting Vehicle Response to Steering and Braking Control Inputs," Highway Safety Research Institute of Science and Technology, The University of Michigan, Michigan, technical report, CST 460, Aug 1969.

[19] M. Kissai, B. Monsuez, A. Tapus , and D. Martinez "A New Linear Tire Model With Varying Parameters," in 2017 International Conference on Automotive and Vehicle Engineering, 2017. In press.

[20] T. A. Johansen and T. I. Fossen, Control Allocation - A survey, in Automatica, Vol. 49, Issue 5, May 2013, pp. 1087?1103.

[21] J. Svendenius, Tire Modeling and Friction Estimation Department of Automatic Control, Lund University, 2007.

[22] O. Harkegard, Efficient active set algorithms for solving constrained least squares problems in aircraft control allocation, Proceedings of the 41st IEEE Conference on Decision and Control, Vol. 2, 2002, pp. $1295-1300$

[23] J. A. M. Petersen and M. Bodson, Constrained quadratic programming techniques for control allocation, in IEEE Transactions on Control Systems Technology, vol. 14, no. 1, pp. 91-98, Jan. 2006.

[24] K.A. Bordignon, Constrained Control Allocation for Systems with Redundant Control Effectors, PhD thesis, Virginia Tech, 1996.

[25] J.J. Burken, P. Lu, Z. Wu, and C. Bahm, Two Reconfigurable FlightControl Design Methods: Robust Servomechanism and Control Allocation, Journal of Guidance, Control, and Dynamics, Vol. 24, No. 3 , pp. 482-493, 2001.

[26] T. A. Johansen, T. I. Fossen, Control allocation ? A survey, Automatica, Volume 49, Issue 5, 2013, Pages 1087-1103.

[27] H. Wallentowitz, Scope for the Integration of Powertrain and Chassis Control Systems: Traction Control - All-Wheel Drive - Active Suspension, in Transportation Electronics, 1990. Vehicle Electronics in the 90's: Proceedings of the International Congress on, 1990, pp. 439-453.

[28] A. T. Van Zanten and R. Bosch Gmbh, Evolution of electronic control systems for improving the vehicle dynamic behavior, in Proceedings of the International Symposium on Advanced Vehicle Control (AVEC), 2002.

[29] Brown G. W., Analysis of 104 Eastern Iowa Motor Vehicle Casualty Accidents, in Proceedings of the Third Triannial Congress on Medical and Related Aspects of Motor Vehicle Accidents. Ann Arbor, Michigan: Highway Safety Research Institute 1971, pp 216 - 218.

[30] V. Ivanov and D. Savitski, Systematization of Integrated Motion Control of Ground Vehicles, in IEEE Access, vol. 3, pp. 2080-2099, 2015.

[31] B. Shyrokau, D. Wang, L. Heidrich and K. Hpping, Analysis of subsystems coordination for electric vehicle during straight-line braking and brake-in-turn, 2013 IEEE Symposium on Computational Intelligence for Engineering Solutions (CIES), Singapore, 2013, pp. 61-67. 